\title{
Degradation of Metalaxyl and Folpet by Filamentous Fungi Isolated From Portuguese (Alentejo) Vineyard Soils
}

\author{
M. Rosário Martins • Pablo Pereira • \\ Nelson Lima · Júlio Cruz-Morais
}

Received: 21 August 2012/Accepted: 29 January 2013/Published online: 22 February 2013

(C) Springer Science+Business Media New York 2013

\begin{abstract}
Degradation of xenobiotics by microbial populations is a potential method to enhance the effectiveness of ex situ or in situ bioremediation. The purpose of this study was to evaluate the impact of repeated metalaxyl and folpet treatments on soil microbial communities and to select soil fungal strains able to degrade these fungicides. Results showed enhanced degradation of metalaxyl and folpet in vineyards soils submitted to repeated treatments with these fungicides. Indeed, the greatest degradation ability was observed in vineyard soil samples submitted to greater numbers of treatments. Respiration activities, as determined in the presence of selective antibiotics in soil suspensions amended with metalaxyl and folpet, showed that the fungal population was the microbiota community most active in the degradation process. Batch cultures performed with a progressive increase of fungicide concentrations allowed the selection of five tolerant fungal strains: Penicillium sp. 1 and Penicillium sp. 2, mycelia sterila 1 and 3, and Rhizopus stolonifer. Among these strains, mycelium sterila 3 and R. stolonifer presented only in vineyard soils treated with repeated application of these fungicides and showed tolerance $>1,000 \mathrm{mg}^{-1}$ against
\end{abstract}

M. R. Martins $(\bowtie) \cdot$ J. Cruz-Morais

Chemistry Department and ICAAM, School of Science and Technology, University of Évora, 7000-671 Évora, Portugal e-mail: mrm@uevora.pt

\section{P. Pereira}

UITA, Unidade de Investigação de Tecnologia Alimentar, INIA, Instituto Nacional de Recursos Biológicos, Campus IAPMEI, Estrada do Paço do Lumiar, 22, 1649-039 Lisboa, Portugal

\section{N. Lima}

Institute for Biotechnology and Bioengineering (IBB), Centre of Biological Engineering, University of Minho, Campus de Gualtar, 4710-057 Braga, Portugal commercial formulations of metalaxyl (10\%) plus folpet (40\%). Using specific methods for inducing sporulation, mycelium sterila 3 was identified as Gongronella sp. Because this fungus is rare, it was compared using csM13polymerase chain reaction (PCR) with the two known species, Gongronella butleri and G. lacrispora. The high tolerance to metalaxyl and folpet shown by Gongronella sp. and R. stolonifer might be correlated with their degradation ability. Our results point out that selected strains have potential for the bioremediation of metalaxyl and folpet in polluted soil sites.

Metalaxyl [methyl $N$-(2,6-dimethylphenyl)- $N$-(methoxyacethyl)-D,L-alaninate] is a systemic acylanilide effective against Oomycetes (Plasmopara viticola Berlese et Toni) that cause downy mildew (Tomlin 1997). This fungicide is a stable compound with good photo-stability (Sukul and Spiteller 2001a), is soluble in water at $20{ }^{\circ} \mathrm{C}\left(7.1 \mathrm{~g} \mathrm{l}^{-1}\right)$, with a vapour pressure of $0.29 \mathrm{mPa}\left(20{ }^{\circ} \mathrm{C}\right)$ and an octanol-water partition coefficient $\left(\mathrm{K}_{\mathrm{ow}}\right)$ of 1.75 (O’Neil et al. 2001). Metalaxyl was classified as a class III-E compound, with an oral $\mathrm{LD}_{50}$ in rats of $669 \mathrm{mg} \mathrm{kg}^{-1}$, and it is considered nontoxic to nontarget arthropod and vertebrate species [United States Environmental Protection Agency (USEPA) United States Environmental Protection Agency 1994]. However, some studies related the genotoxic and mutagenic effects of metalaxyl (Hrelia et al. 1996). Because of its low adsorption and high mobility, metalaxyl lixiviated easily in soil and can contaminate groundwater, so its residues are a serious environmental threat (Andrades et al. 2001; Sukul 2006; Martin et al. 2012; Wightwick et al. 2012). Indeed, metalaxyl residues have been found in groundwater at concentrations $\leq 0.49 \mu \mathrm{g}^{-1}$, which exceeds the $0.1 \mu \mathrm{g} 1^{-1}$ European Union limit (Hildebrandt et al. 2008). 